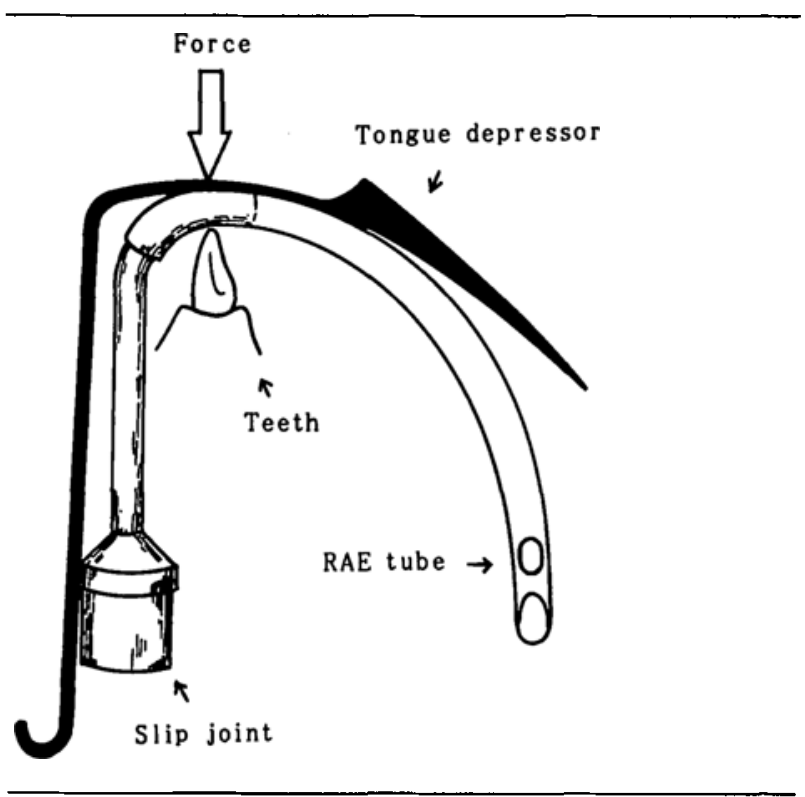

FIGURE 2 The slip joint prevents compression of the RAE tube between tongue depressor and lower incisor teeth.

2). In addition, the slip joint may prevent herniation, obstruction, and kinking of the RAE tube. ${ }^{1,2,4}$

K. Sugiyama DDS PhD

K. Yokoyama DDS PhD

Y. Satoh DDS

Department of Anesthesia

Kagoshima University Dental Hospital

8-35-1 Sakuragaoka, Kagoshima 890, Japan

REFERENCES

1 Sen AK, Gupta B. Herniation of RAE tube through split Boyle Davis gag (Letter). Anaesth Intensive Care 1990; 18: 577-8.

2 Graham D, Kaddour HS. RAE tube obstruction during tonsil dissection (Letter). Br J Anaesth 1996; 76: 170-1.

3 Sun $K O$. Prevention of bronchial intubation with RAE tube during adenotonsillectomy (Letter). Anaesthesia 1993; 48: 645 .

4 Yamashita $M$, Motokawa K. Preventing kinking of disposable preformed endotracheal tubes (Letter). Can J Anaesth 1987; 34: 103

\section{Epidural pressure in a patient with superior vena cava syndrome}

To the Editor:

We present a patient in whom the cervical epidural pressure and the extent of epidural analgesia increased after obstruction of the superior vena cava (SVC). In a 76-yr-old man, who suffered from burning pain in his right hand and forearm, an epidural catheter was inserted at the $C_{7}-T_{1}$ interspace and epidural blockade was performed for pain relief due to reflex

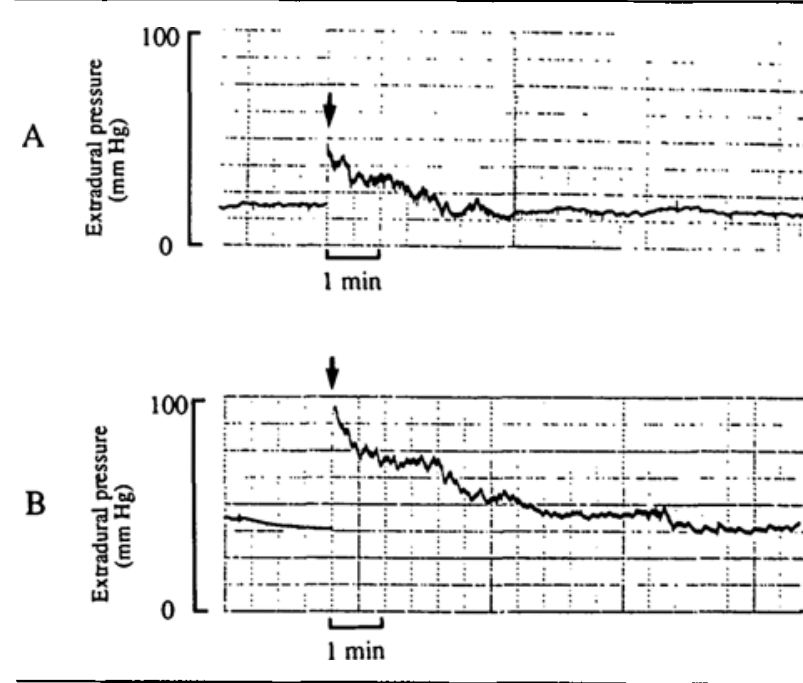

FIGURE The basal epidural pressure was $18 \mathrm{mmHg}$ and after epidural injection of $6 \mathrm{ml}$ of bupivacaine $0.25 \%$ (arrow) it increased to $36 \mathrm{mmHg}$ before SVC obstruction (on admission) (A); after SVC obstruction (6th hospital day), the basal pressure was elevated (38 $\mathrm{mmHg}$ ) and the injection of the same dose of bupivacaine increased it to $98 \mathrm{mmHg}(\mathrm{B})$.

sympathetic dystrophy (RSD). The SVC syndrome abruptly occurred on the sixth hospital day: the SVC was completely obstructed by an apical lung tumour and iv thrombosis, suggesting that his pain was caused by brachial plexus pathology due to the tumour and not by RSD. The epidural pressures were measured through the catheter in the supine position. After SVC obstruction, the basal epidural pressure was elevated: epidural injection of $6 \mathrm{ml}$ bupivacaine $0.25 \%$ considerably increased the pressure (Figure) and induced severe headache and nausea. The extent of analgesia was $\mathrm{C}_{4}-\mathrm{T}_{4}$ and $\mathrm{C}_{4} \mathrm{~T}_{10}$ before and after the obstruction, respectively.

Superior vena caval obstruction increases epidural and intracranial pressures (ICP). Since an epidural volume load increases ICP, especially in patients with increased ICP, ' the epidural injection of bupivacaine would produce an increase in ICP after SVC obstruction. Furthermore, SVC obstruction may unexpectedly increase the extent of epidural analgesia, as was seen in the present patient, probably due to the decreased volume of the epidural space by venous dilatation.

Mikito Kawamata MD

Keiichi Omote MD

Shinzoh Sumita MD

Hiroshi lwasaki MD

Akiyoshi Namiki MD PhD

Department of Anesthesiology

Sapporo Medical University

South-1, West-16, Chuo-ku

Sapporo 060, Japan

\section{REFERENCE}

1 Hilt $H$, Gramm HJ, Link J. Changes in intracranial pressure associated with extradural anaesthesia. $\mathrm{Br} \mathrm{J}$ Anaesth 1986; 58: 676-80. 